\title{
Optical photon transport in powdered-phosphor scintillators. Part 2. Calculation of single-scattering transport parameters.
}

\author{
Gavin G. Poludniowski
}

Joint Department of Physics, Division of Radiotherapy and Imaging, Institute of Cancer

5 Research and Royal Marsden NHS Foundation Trust, Downs Road, Sutton, Surrey, SM2

$5 P T, U K$

Philip M. Evans

Centre for Vision Speech and Signal Processing (CVSSP), Faculty of Engineering and Physical Sciences, University of Surrey, Guildford, Surrey, GU2 7XH

\section{ABSTRACT}

Purpose: Monte Carlo methods based on the Boltzmann transport equation have previously been used to model light transport in powdered-phosphor scintillator screens. Physically-motivated guesses or, alternatively, the complexities of Mie theory have been used by some authors to provide the necessary input transport parameters. The purpose of Part 2 of this work is to: (I) investigate the impact of size-distribution and emission spectrum on Mie predictions of transport parameters; (II) suggest simpler geometrical optics-based models and compare the predictions to Mie theory; (III) validate the optics-based predictions of transport parameters using Modulation Transform Function (MTF) data published for two $\mathrm{Gd}_{2} \mathrm{O}_{2} \mathrm{~S}: \mathrm{Tb}$ screens..

Methods: The following transport parameters are calculated: scattering efficiency $\left(Q_{s c t}\right)$, absorption efficiency $\left(Q_{a b s}\right)$ and the scatter anisotropy $(g)$. Calculations assume spherical phosphor grains and are performed using the analytic method of Mie theory for grain radii of 0.1 to $5.0 \mu \mathrm{m}$. The sensitivity of transport parameters to emission wavelength is investigated using an emission spectrum representative of that of $\mathrm{Gd}_{2} \mathrm{O}_{2} \mathrm{~S}: \mathrm{Tb}$. The impact of a grain-size distribution in the screen on the parameters is investigated using a Gaussian size-distribution ( $\sigma=1 \%, 5 \%$ or $10 \%$ of mean radius). 
25 Two simple approximations for the calculation of the transport parameters are suggested: a geometrical optics and diffraction model (GODM) and an extension of this (GODM+). Comparisons to measured MTF are made for two commercial screens: Lanex Fast Back and Lanex Fast Front (Eastman Kodak Company Inc., USA).

Results: The Mie theory predictions of transport parameters are shown to be highly sensitive to both grain size and emission wavelength. For a phosphor screen structure with a distribution in grain sizes and a spectrum of emission, only the average trend of Mie theory is likely to be important. This average-behavior is well predicted by the more sophisticated of the geometrical optics models $(\mathrm{GODM}+)$ and in approximate agreement for the simplest (GODM). The agreement of MTF predictions with experiment is reasonable, and encouraging given the uncertainties in screen composition.

Conclusion: If Mie theory is used for calculating transport parameters for light scattering and absorption in powdered-phosphor screens, care should be taken to average out the fine-structure in the parameter predictions. However, for visible emission wavelengths $(\lambda<1.0 \mu \mathrm{m})$ and grain radii $(a>$ $1.0 \mu \mathrm{m})$, geometrical optics models for transport parameters are a reasonable alternative to Mie theory. Their greatest virtue is simplicity and the results presented suggest no substantial loss in predictive accuracy.

\section{INTRODUCTION}

In many medical $\mathrm{x}$-ray imaging devices indirect-detection of $\mathrm{x}$-rays is used to increase the detective quantum efficiency of the imaging system. A commonly used technology is that of a powderedphosphor screen. In such a screen, grains of a phosphor are packed within an inert binder material. ${ }^{1} \mathrm{X}$ rays or secondary electrons interact in a screen and result in the subsequent emission of optical photons. These are collected at a photo-sensor and provide an "indirect" detection of the incident xrays. Powder-phosphor screens have been used widely in medical imaging as discussed in Part I of this work. ${ }^{2}$ Despite the quantum gain from the use of powdered-phosphors, light scattering and 
absorption in the screen leads to a degradation in the modulation-transfer-function (MTF) and lightcollection efficiency. This depends on the geometry and structure of the screen and there is interest in characterizing and optimizing such effects (see references to Part 1 for further details).

In Part 1 the use of the Boltzmann transport equation (BTE) to model scattering and absorption in a phosphor screen was validated against an explicit microscopic treatment. The BTE approach is much more efficient than the explicit treatment of a screen granular microstructure. The phosphor and binder are treated as a homogeneous scattering medium. It was shown that the high packing densities in phosphor screens do not invalidate the BTE approach. A Monte Carlo model based on the BTE needs, as inputs, the transport parameters for scattering of an optical photon from a single grain. ${ }^{3}$ Three independent parameters are essential for input into a BTE Monte Carlo: the scattering efficiency $\left(Q_{s c t}\right)$, absorption efficiency $\left(Q_{a b s}\right)$ and scattering anisotropy factor $(g)$. These are generally unknown or imprecisely known from experiment. Some authors have simply made physicallymotivated "guesses" for them. For example: $Q_{s c t}$ has been calculated based on the average inter-grain spacing and isotropic scatter assumed $(g=0) .{ }^{4}$ Working backward and inferring the parameters by comparison of ensembles of simulations with experiment has also been tried. ${ }^{5}$ The capability of theoretically calculating the transport parameters is desirable. In this work, Part 2, we examine models for such calculations. The accuracy of geometrical optics for calculating transport parameters is assessed in comparison to the more sophisticated approach of Mie theory. Mie theory predicts the necessary parameters from first principles, given the grain size, light wavelength and the refractive indices (RIs) of the binder and phosphor. ${ }^{6}$ The application of Mie theory to powdered-phosphor materials dates back to at least the $1970 \mathrm{~s},{ }^{7}$ but its incorporation into models in the field of medical imaging appears much more recent. ${ }^{3,8-10}$ While a powerful approach, the use of Mie theory to model phosphor screens has limitations. ${ }^{6}$ In particular:

(i) Mie theory is only a solution for perfect spheres and phosphor granules are irregular and vary in size and shape; 
(ii) Its predictions have fine-structure that is unrealistically numerically sensitive; the precise values predicted can vary greatly with a small change in grain size or emission wavelength;

(iii) The form of the Mie formulae is complicated involving series summations over oscillating Riccati-Bessel functions.

Geometrical optics provides an alternative framework for approaching the problem. Numerical

calculations of transport parameters are performed here for the common $\mathrm{Gd}_{2} \mathrm{O}_{2} \mathrm{~S}: \mathrm{Tb}$ (GOS:Tb) phosphor, to assess the impact of grain size distribution, emission spectrum and various approximations (Mie, geometrical optics-based) on predictions. Two varieties of Lanex screens (Eastman Kodak Company Inc., USA) are simulated and predictions of the MTFs are compared to experimental measurements. ${ }^{11}$

\section{METHOD AND MATERIALS}

\section{II.A Phosphor screen model}

The grains of a powdered phosphor are embedded in an inert binder material to form a phosphor layer in a screen. Optical photons emitted within the grains undergo scattering as they propagate through the layer, due to mismatches in the RIs between the grain and the surrounding binder. The complex RI of a grain is denoted,

$$
m_{g}=n_{g}-i z_{g}
$$

where the degree of absorption depends on the small imaginary component. Following previous authors, ${ }^{3}$ the value $m_{g}=2.3-i 10^{-6}$ is used for GOS:Tb. A polyurethane elastomer is used in Lanex screens. This binder's RI is assumed to be real and the value chosen is $n_{b}=1.5$. This estimate is based on typical values for such binders $(1.4<\mathrm{RI}<1.6) .{ }^{12}$ The relative phosphor-to-binder relative refractive indices will be defined as,

$$
n=\frac{n_{g}}{n_{b}} ; \text { and } z=\frac{z_{g}}{n_{b}}
$$


The four main emission peaks of GOS:Tb are presented in Table 1 along with their relative intensities. ${ }^{13}$ An approximation to the full spectrum was constructed by representing it as four Gaussian peaks with a Full-Width-Half-Maximum (FWHM) of $0.015 \mu \mathrm{m}$. This FWHM was arrived at by inspection of published spectra. ${ }^{13,14}$ The resulting spectrum is depicted in Fig. 1.

Table 1. The peak wavelength and relative intensities for the emissions of GOS:Tb. Figures based on sample GOS14 in Ref. 13.

\begin{tabular}{lcccc}
\hline \hline Wavelength, $\lambda[\mu \mathrm{m}]$ & 0.489 & 0.545 & 0.586 & 0.619 \\
\hline Relative intensity [A.U.] & 1.00 & 2.80 & 0.44 & 0.19 \\
\hline \hline
\end{tabular}

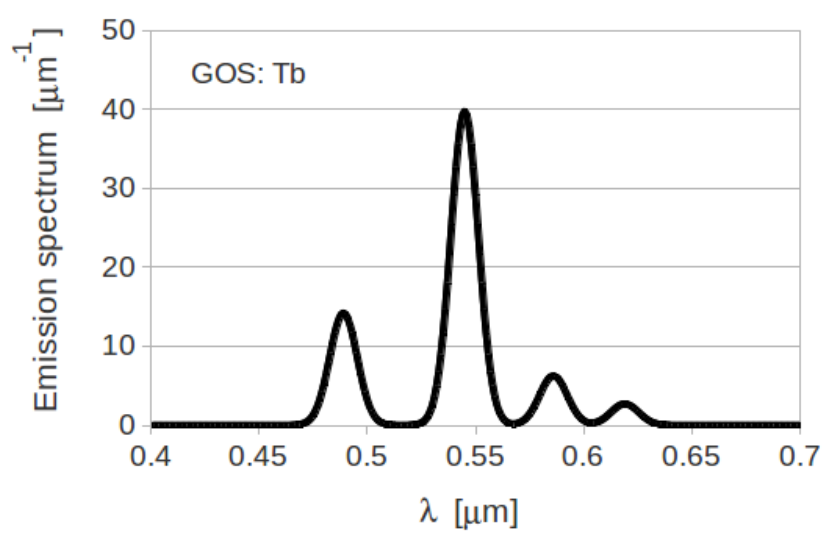

FIG 1. Approximation to the emission spectrum of GOS:Tb.

It is standard 6 to describe scattering and absorption behavior in terms of a "size-parameter", $x$, where

$$
x=\frac{2 \pi n_{b}}{\lambda} a
$$

and $\lambda$ is the photon wavelength and $a$ is the grain radius. Geometrical optics describes behavior in the

110 limit where $x>>1$. The average grain radius in commercial phosphor screens typically corresponds to the range 1 to $5 \mu \mathrm{m} .{ }^{15,16}$ An examination of Table 1 and these values of of grain size indicate that in typical phosphor screens, the size-parameter is in fact substantially greater than unity.

\section{B Transport parameters}


The efficiency factor for an interaction, $i$, is the ratio of an interaction cross-section to the physical projected area of the grain: ${ }^{6}$

$$
Q_{i}=\frac{C_{i}}{A}=\frac{C_{i}}{\pi \mathrm{a}^{2}}
$$

where $C_{i}$ is the interaction cross-section, $A$ is the projected area and $a$ is the grain radius. If there is a distribution in grain sizes and that distribution is known, a size-averaged efficiency can be calculated. The averaged efficiency is,

$$
Q_{i}=\frac{\int_{0}^{\infty} n(a) C_{i}(a) d a}{\pi \int_{0}^{\infty} n(a) a^{2} d a}
$$

where $n(a)$ is the probability function for grain size. The effects of a Gaussian distribution, which describes the grain size distribution in some screens, ${ }^{13}$ is investigated. Other distributions would be expected to have a similar averaging effect. The spread, $\sigma$, as a percentage of the mean radius, was set to $1 \%, 5 \%$ or $10 \%$. Three efficiency factors (and hence interaction lengths) are relevant: the absorption $\left(Q_{a b s}\right)$, the scattering $\left(Q_{s c t}\right)$ and the extinction $\left(Q_{e x t}\right)$. The last of these is the sum of the first two. The interaction length for such an interaction process $i$,

$$
l_{i}=\frac{V}{A} \frac{1}{p_{f} Q_{i}}=\frac{4}{3} \frac{a}{p_{f}} \frac{1}{Q_{i}},
$$

where $V$ is the grain volume and $p_{f}$ is the packing fill fraction.

A further transport parameter, the anisotropy factor, $g$, can be used to parameterize the angular scattering distribution. Such a parameterization is needed, in a Monte Carlo transport code, to decide the outgoing direction of an optical photon following a simulated scatter event (see Part $1^{2}$ ). The Henyey-Greenstein distribution is used here: 


$$
P_{H G A}\left(\boldsymbol{\Omega}, \boldsymbol{\Omega}^{\prime}\right)=\frac{1}{4 \pi} \frac{1-g^{2}}{\left(1+g^{2}-2 g \boldsymbol{\Omega} \cdot \boldsymbol{\Omega}^{\prime}\right)^{3 / 2}} \text { where } \int_{4 \pi} P_{H G A}\left(\boldsymbol{\Omega} \cdot \boldsymbol{\Omega}^{\prime}\right) d \boldsymbol{\Omega}=1
$$

and where $\boldsymbol{\Omega}$ is the initial optical photon direction and $\boldsymbol{\Omega}$ is the scattered direction.

\section{II.C Mie theory transport parameters}

Mie theory is a rigorous solution to Maxwell's equations for the following problem: scattering and absorption of an electromagnetic plane-wave from a homogeneous sphere. Mie theory is applicable in principle, for all value of $x$, although in practice there may be numerical limitations for very large $x .^{17}$ The Mie formulae are, ${ }^{6}$

$$
\begin{aligned}
& Q_{e x t}^{\text {Mie }}=\frac{2}{x} \sum_{n=1}^{\infty}(2 n+1) \operatorname{Re}\left(a_{n}+b_{n}\right), \\
& Q_{s c t}^{\text {Mie }}=\frac{2}{x} \sum_{n=1}^{\infty}(2 n+1)\left(\left|a_{n}\right|^{2}+\left|b_{n}\right|^{2}\right),
\end{aligned}
$$

and

$$
\overline{\Omega \cdot \Omega^{\prime}} Q_{s c t}^{M i e}=\frac{4}{x^{2}} \sum_{n=1}^{\infty} \frac{n(n+2)}{n+1} \operatorname{Re}\left(a_{n} a_{n+1}^{*}+b_{n} b_{n+1}^{*}\right)+\frac{4}{x^{2}} \sum_{n=1}^{\infty} \frac{2 n+1}{n(n+1)} \operatorname{Re}\left(a_{n} b_{n}^{*}\right),
$$

where

$$
a_{n}=\frac{\psi_{n}{ }^{\prime}(m x) \psi_{n}(x)-m \psi_{n}(m x) \psi_{n}{ }^{\prime}(x)}{\psi_{n}{ }^{\prime}(m x) \zeta_{n}(x)-m \psi_{n}(m x) \zeta_{n}{ }^{\prime}(x)}
$$

and

$$
b_{n}=\frac{m \psi_{n}{ }^{\prime}(m x) \psi_{n}(x)-\psi_{n}(m x) \psi_{n}{ }^{\prime}(x)}{m \psi_{n}{ }^{\prime}(m x) \zeta_{n}(x)-\psi_{n}(m x) \zeta_{n}{ }^{\prime}(x)}
$$

The special functions $\psi_{n}$ and $\zeta_{n}$ are the Riccati-Bessel functions and the prime indicates a derivative with respect to the argument. These functions can be calculated from well-known recurrence 
relationships. ${ }^{17}$ The quantity $g=\overline{\Omega \cdot \Omega^{\prime}}$ is the mean cosine of scattering angle. It has been shown that the infinite series converge after a set number of terms (e.g. $n_{\max }=x+4 x^{1 / 3}+2$ ). The summation of further terms will eventually lead to numerical instabilities and therefore the solution is truncated at such a value. The Mie formulae were coded in Fortran 95 and predictions validated against tabulated values. ${ }^{17}$

\section{II.D Geometrical optics transport parameters}

In Part 1 of this work $^{2}$ a geometrical optics model was used to calculate the required transport parameters. This model will be formulated slightly differently in Part 2. The reason for this is that Mie scattering, as an exact solution to Maxwell's equations, includes diffraction contributions ${ }^{6}$ and a direct comparison to Mie theory is desirable. Therefore diffraction will be included in a geometrical optics and diffraction model (GODM) in a well-established manner. ${ }^{6}$

The part of a plane-wave wave-front that is incident on the projected area of a phosphor grain can be treated as a set of parallel rays obeying geometrical optics: each ray is either refracted/reflected or absorbed by the grain. All this energy is therefore lost from the initial beam: $Q_{e x t}^{G O M}=1$. However, the outgoing wave-front, beyond the grain, will have a "hole" in it. The consequence of this is that interference occurs in the far-field (diffraction) which further diminishes the energy in the original beam. Note that the projected area of a spherical grain is a circle. A phosphor grain therefore resembles an opaque disc. The scattering of a plane-wave from an opaque disc is a classic (and solved) wave-optics problem. Babinet's principle dictates that the extinction cross-section of a diffracting body is twice its geometric area. ${ }^{6}$ That is: $Q_{e x t}^{D}=Q_{s c t}^{D}=1$. This provides the result,

$$
Q_{\text {ext }}^{G O D M}=Q_{\text {ext }}^{G O M}+Q_{\text {ext }}^{D} \cong 2
$$

Unlike in Mie theory, the absorption efficiency can be calculated directly by summation of all orders of reflection and refraction. Equivalently to equation (18) in Part 1 it takes the form, 


$$
Q_{a b s}^{G O D M} \cong \frac{16 \pi}{3} \frac{a n_{b}}{\lambda} z F(n)
$$

where for weakly absorbing spheres, $F(n)=n^{2}\left(1-\left(1-n^{-2}\right)^{3 / 2}\right) .^{18}$

175 It is useful to consider separate anisotropy factors for both contributing components: $g^{G O M}$ and $g^{D}$. Diffraction is tightly forward focused, and, in our approximation, $g^{D}=1$. This means that diffraction is indistinguishable from the original beam and could be neglected entirely. The form of $g^{G O M}$ is known: ${ }^{6,19}$

$$
g^{G O M}=\frac{1}{2} \sum_{i=1,2} \int_{0}^{1} \frac{2 r_{i}^{4}\left(1-\cos 2 \tau^{\prime}\right) \cos 2 \tau+\left(1-r_{i}^{2}\right)^{2} \cos \left(2 \tau-2 \tau^{\prime}\right)}{1-2 r_{i}^{2} \cos 2 \tau^{\prime}+r_{i}^{4}} d\left(\cos ^{2} \tau\right)
$$

where

$$
r_{1}=\frac{\sin \left(\tau-\tau^{\prime}\right)}{\sin \left(\tau+\tau^{\prime}\right)}, r_{2}=\frac{\tan \left(\tau-\tau^{\prime}\right)}{\tan \left(\tau+\tau^{\prime}\right)} \text { and } \cos \tau^{\prime}=\frac{1}{n} \cos \tau
$$

This formulation is rather cumbersome. The authors, however, have found it readily parameterized by a simple exponential such that, $g^{G O M} \approx \exp (-\alpha(n-1))$. The value $\alpha=0.86$ provided a good fit to that found by numerical integration $\left(R^{2}=0.999\right)$. Equation $(8 \mathrm{c})$, evaluated by numerical integration, is compared with the exponential approximation in Fig. 2. This results in the following form for the GODM anisotropy factor:

$$
g^{G O D M}=\frac{g^{G O M} Q_{s c t}^{G O M}+g^{D} Q_{s c t}^{D}}{Q_{s c t}^{G O M}+Q_{s c t}^{D}} \cong \frac{1+g^{G O M}}{2} \cong \frac{1+e^{-\alpha(n-1)}}{2} .
$$




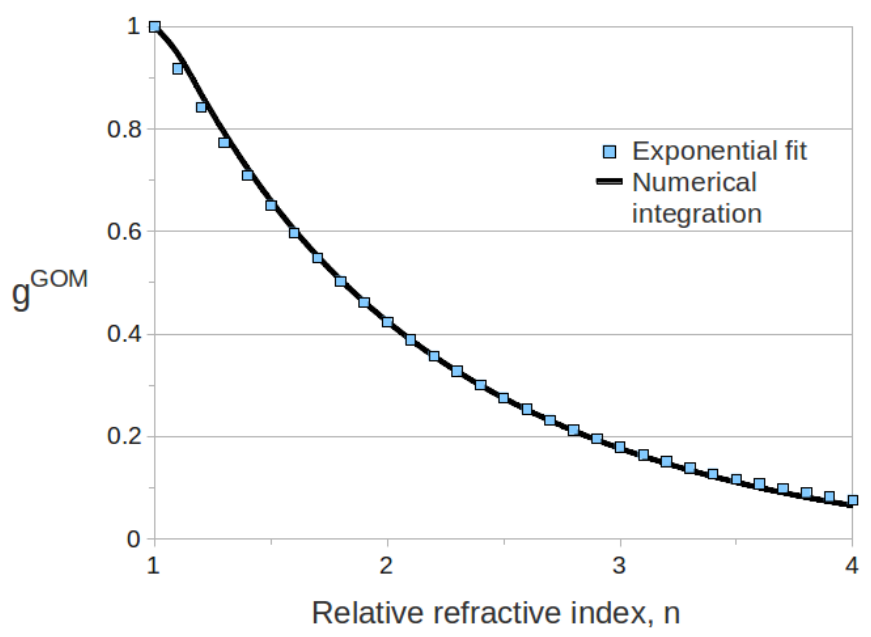

FIG. 2. The exponential approximation to the geometrical optics anisotropy factor, $g^{G O M}$, compared to the exact geometrical optics results found by numerical integration.

\section{II.E Addition of edge effects}

Despite the treatment outlined above a phosphor grain is, in fact, not an opaque disc. A grain is a three-dimensional object with a two-dimensional surface. The curvature of the sphere at grazing incidence produces effects that modify the diffraction. ${ }^{6}$ Further, geometrical optics and ray-tracing are known to be inaccurate at grazing incidence. ${ }^{6}$ These two sources of inaccuracy will be referred to as "edge-effects". The result is that, for large but finite $x$, the GODM is known to be inexact. Mie theory, however, is an exact solution to Maxwell's equations. Fortunately, the asymptotic form of the Mie extinction efficiency is known, in the limit $x \rightarrow \infty$. To the leading two terms in $x$, it has been shown to $b^{20}$

$$
Q_{e x t}^{G O D M+}=Q_{e x t}^{G O D M}\left(1+x^{-2 / 3}\right) \cong 2\left(1+x^{-2 / 3}\right),
$$

where we denote the extended model GODM+. No intuitive explanation can be provided for the form of the additional decaying component. This asymptotic contribution can, however, be identified with the edge-effects discussed above. The scattering and absorption efficiencies also have dependencies on $x^{-2 / 3}$, but not such a elementary form. ${ }^{20}$ However, the simplest modification consistent with weak absorption i.e. $Q_{s c t}^{G O D M+} \cong Q_{e x t}^{G O D M+}$, suggests, 


$$
Q_{s c t}^{G O D M+}=Q_{s c t}^{G O D M}\left(1+x^{-2 / 3}\right)
$$

and hence

$$
Q_{a b s}^{G O D M+}=Q_{a b s}^{G O D M}\left(1+x^{-2 / 3}\right) \cong \frac{16 \pi}{3} \frac{a n_{b}}{\lambda} z F(n)\left(1+x^{-2 / 3}\right) .
$$

We then suggest that the anisotropy be parameterized as,

210

$$
\begin{gathered}
g^{G O D M+}=\frac{g^{G O D M} Q_{s c t}^{G O D M}+g^{+}\left(Q_{s c t}^{G O D M+}-Q_{s c t}^{G O D M}\right)}{Q_{s c t}^{G O D M+}} \\
\cong \frac{1}{2}\left(1+e^{-\alpha(n-1)}+2 g^{+} x^{-2 / 3}\right)\left(1+x^{-2 / 3}\right)^{-1}
\end{gathered}
$$

where $g^{+}$is the anisotropy associated with the edge component. Comparison with the predictions of Mie theory suggested that an empirical value of, $g^{+}=0.25$, is adequate for GOS:Tb.

\section{II.B Lanex Fast screens}

MTFs were simulated for two commercial GOS:Tb phosphor screens: Lanex Fast Front and Lanex Fast Back. The experimental measurements used in this study were taken from Ref. 11. In that study a RadEye CMOS detector (Rad-Icon Imaging Corp., USA) was used in direct contact with a screen. The residual air gap between screen and detector surface was minimized by screwing a $1 \mathrm{~mm}$ thick graphite compression plate down on top of the screen. ${ }^{11,21} \mathrm{~A}$ thin polyurethane foam sheet was inserted between the graphite and screen. The layers of apparatus are illustrated schematically in Fig. 3. Following a related study, ${ }^{2}$ we assume a thin layer of air $(\approx 1 \mu \mathrm{m})$ to be trapped between the screen lower surface and the detector. Note that no optical coupling fluid appears to have been utilized. ${ }^{21}$ The experimental MTF measurements were obtained with a $70 \mathrm{kVp}$ x-ray beam of HVL $7.1 \mathrm{~mm} \mathrm{Al}$ and a line-spread method.

The approximate structure of the Lanex Fast Back screen is presented in Table 2. ${ }^{11}$ The polyethylene terephthalate $(\mathrm{PET})$ support also contains $\mathrm{TiO}_{2}$ particles providing a reflectivity of $\approx 0.88$ at that surface. The Lanex Fast Front screen will be assumed to have the same structure but a reduced 
phosphor thickness of $110 \mu \mathrm{m} .{ }^{11}$ The dose-deposition in the two screens was calculated using the DOSRZnrc (EGSnrc) Monte Carlo code ${ }^{23}$ and a narrow pencil beam of radius $1 \mu \mathrm{m}$. All interaction processes were turned on in EGSnrc (notably atomic relaxations). A $70 \mathrm{kVp}$ input $\mathrm{x}$-ray spectrum with a HVL matching the experimental beam was generated using the freely available SpekCalc program. ${ }^{24}$ Simulations used $10^{7} \mathrm{x}$-ray histories per screen. Dose was scored in the phosphor layer in DOSRZnrc for radial bins up to $10 \mathrm{~cm}$. The phosphor layer was also sub-divided into either $1 \mu \mathrm{m}$ (Lanex Fast Front) or $2 \mu \mathrm{m}$ (Lanex Fast Back) sub-layers for scoring purposes. The four components of the screen as listed in Table 1 were included in the Monte Carlo as well as the graphite plate and polyurethane (PU) foam. The PU foam was estimated to have a thickness of $1 \mathrm{~mm}$ and a density of $0.05 \mathrm{~g} \mathrm{~cm}^{-3}$ (the actual values were not available). The scored dose distributions in the phosphor layer were used as the spatial-distributions for subsequent optical photon emission.

Optical transport in the screens was conducted using the Boltzmann transport equation Monte Carlo code as described in Part 1 of this work. ${ }^{2}$ The grain radius in the Lanex fast screens was assumed to be $4.25 \mu \mathrm{m}$. This corresponds to the middle of the range of median diameters (8 to $9 \mu \mathrm{m})$ inferred from the patent literature for Eastman Kodak high-speed screens. ${ }^{15}$ The packing fill-factor, $p_{f}$, based on nominal phosphor thickness and density, was set to 0.6 (see Table 2). The binder in the Lanex screens (a polyurethane elastomer) was assumed to have a RI, $n_{b}=1.5$, and the complex RI of GOS:Tb was taken as, $m_{g}=2.3-i 10^{-6}$, as described in Section II.A. Optical photons incident at the upper 245 phosphor-PET interface of the screen were assumed (specularly) reflected with a probability 0.88 and were otherwise stopped. The thin protective coating at the bottom of the screen was assumed to be matched to the binder in RI, as the RI of cellulose acetate is $\approx 1.5 .{ }^{25}$ Optical photons reaching the phosphor-protective coating interface thus propagated into the cellulose acetate undeviated. However, upon reaching the outer surface of the coating, the optical photon could be reflected back due to the residual air interface. The spatial distribution of optical photons exiting the protective coating was assumed to correspond to the pre-sampled MTF at the active region of the detector. The pre-sampled MTF was calculated from the simulated histories as follows (see Part 1): 


$$
\operatorname{MTF}(w)=\frac{1}{D} \sum_{i=1}^{D} J_{0}\left(2 \pi \rho_{i} w\right)
$$

where $D$ is the number of detected photons, $\rho_{i}$ is its lateral displacement and $w$ is the spatial frequency (cycles/mm). Note that the pixel-size of the CMOS detector $(48 \mu \mathrm{m})$ was small-enough that the sinc function contribution to MTF associated with sampling pitch could be neglected for the spatial-frequencies of interest $\left(0-5\right.$ cycles $\left.\mathrm{mm}^{-1}\right){ }^{11}$

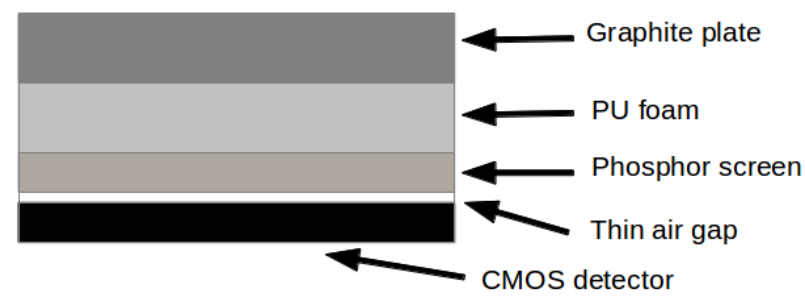

FIG 3. Illustration of the measurement configuration used in Refs. 8 and 18.

Table 2. Structure of Lanex Fast Back screen.

\begin{tabular}{llll}
\hline \hline Material & Function & Thickness $[\mu \mathrm{m}]$ & Density $\left[\mathrm{g} \mathrm{cm}^{-3}\right]$ \\
\hline Cellulose acetate & Anti-curl layer & $55^{*}$ & $1.44^{\dagger}$ \\
PET & Support & $275^{*}$ & $1.38^{\dagger}$ \\
Phosphor (+ binder) & Scintillator & $300^{*}$ & $4.48^{*}$ \\
Cellulose acetate & Protective overcoat & $13^{*}$ & $1.44^{\dagger}$ \\
\hline \hline
\end{tabular}

*Values taken from Ref. 11. ${ }^{\dagger}$ Values taken from the default values in the pegs4dat data of EGSnrc. ${ }^{23}$

\section{RESULTS}

Fig. 4(a) to 4(c) show the transport parameters $\left(Q_{e x t}, Q_{a b s}, g\right)$ as predicted by Mie theory at an emission wavelength of $0.545 \mu \mathrm{m}$. Note the erratic fine-structure in predictions (for all three parameters) and the smoother oscillations about the average trend (for $Q_{e x t}$ and $g$ ). Also depicted are the predictions when averaging is performed over the full GOS:Tb emission spectrum defined in II.A. The averaging over wavelength has a smoothing effect on the fine-structure and oscillations. This smoothing effect is relatively weak, since the GOS:Tb emission spectrum is narrow, compared to 
some scintillators e.g. CsI. ${ }^{14}$ Note that the wavelength-averaged predictions follow the same underlying trend as for emissions at $0.545 \mu \mathrm{m}$.

Fig. 5(a) to 5(c) show results for Mie theory and Gaussian grain-size distributions of various spreads ( $\sigma=1 \%, 5 \%$ and $10 \%$ ), for optical emissions at the peak GOS:Tb wavelength of $0.545 \mu \mathrm{m}$. Even a very small distribution in grain sizes $(\sigma=1 \%)$ results in the removal of most fine-structure observed in Fig. 4(a) to Fig.4(c). A broader distribution of $\sigma=5 \%$ also produces an appreciable dampening of the oscillations about the underlying trends. By a moderate size spread of $\sigma=10 \%$, the oscillations about the trend values are almost entirely absent for grain radii $>1 \mu \mathrm{m}$.

Fig. 6(a) to 6(c) show the predictions of the geometrical optics-based models (GODM and GODM+), in comparison to Mie theory averaged over wavelength and (very narrow) size-distribution $(\sigma=1 \%)$. The GODM model gives approximately correct values. However, it underestimates the Mie prediction trends of $Q_{e x t}$ and $Q_{a b s}$ by $\approx 5 \%$ and $30 \%$, respectively. The asymmetry parameter, $g$, is overestimated by $\approx 3 \%$ at larger radii. The extended model (GODM+) substantially improved agreement for both $Q_{e x t}$ and $g$. Improvement for $Q_{a b s}$ was slight, however. The general agreement of the GODM and GODM+ models with average Mie behavior justifies their use as inputs into transport calculations.

285 Fig. 7(a) presents the results of the DOSRZnrc Monte Carlo calculations for the distribution of relative dose with depth (summed over radial dimension) in Lanex Fast Front and Fast Back screens. Decay in dose-deposition with depth in the phosphor layer is clear, but the effect of electron disequilibrium close to interfaces with neighboring layers is also apparent. Fig. 8(b) shows the radial distribution (summed over depth) for both screens. A rapid fall-off is exhibited with radial displacement. The peak normalizing dose in the central scoring zone is that averaged over a $10 \mu \mathrm{m}$ radius cylinder.

Using the three-dimensional dose-distributions calculated with DOSRZnrc and the optical transport Monte Carlo code, MTFs were simulated. They are presented in Fig. 8(a). The input transport 
parameters $\left(Q_{e x t}, Q_{a b s}, g\right)$ were calculated for both the GODM and GODM+ models (and emission at $0.545 \mu \mathrm{m})$. Both models give substantial agreement with experimental MTF measurements for both screens. Note that no fitting was performed: the simulated MTFs were calculated using the best estimates of the various physical parameters available. The precise dose-distribution resulting from the $\mathrm{x}$-ray beam e.g. scatter and atomic relaxations was observed to have a quantitative impact on the resulting MTF, although this was of secondary importance to optical diffusion for the two screens and x-ray beam quality examined
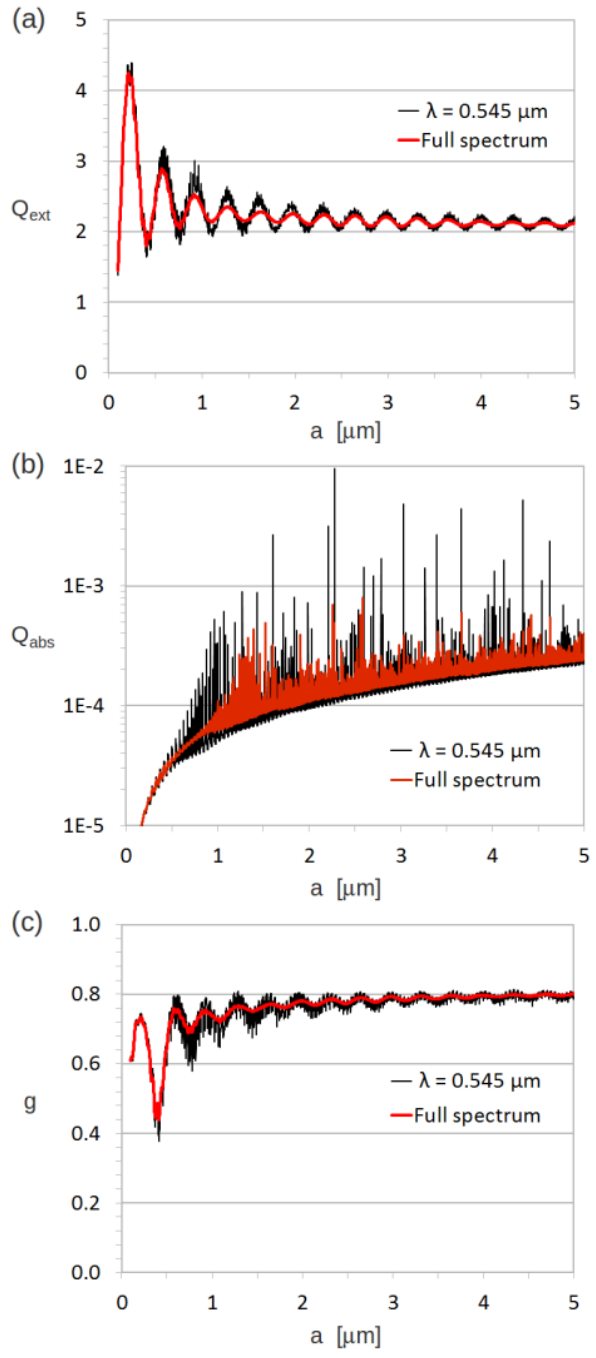

FIG. 4. Mono-disperse Mie theory calculations for GOS:Tb spheres in binder for emissions at $0.545 \mu \mathrm{m}$ and averaged over GOS:Tb emission spectrum. Shown as a function of radius, $a$, are: (a) the extinction efficiency, $Q_{e x t}(\mathrm{~b})$ the absorption efficiency, $Q_{a b s}$ and (c) the asymmetry factor, $g$. 

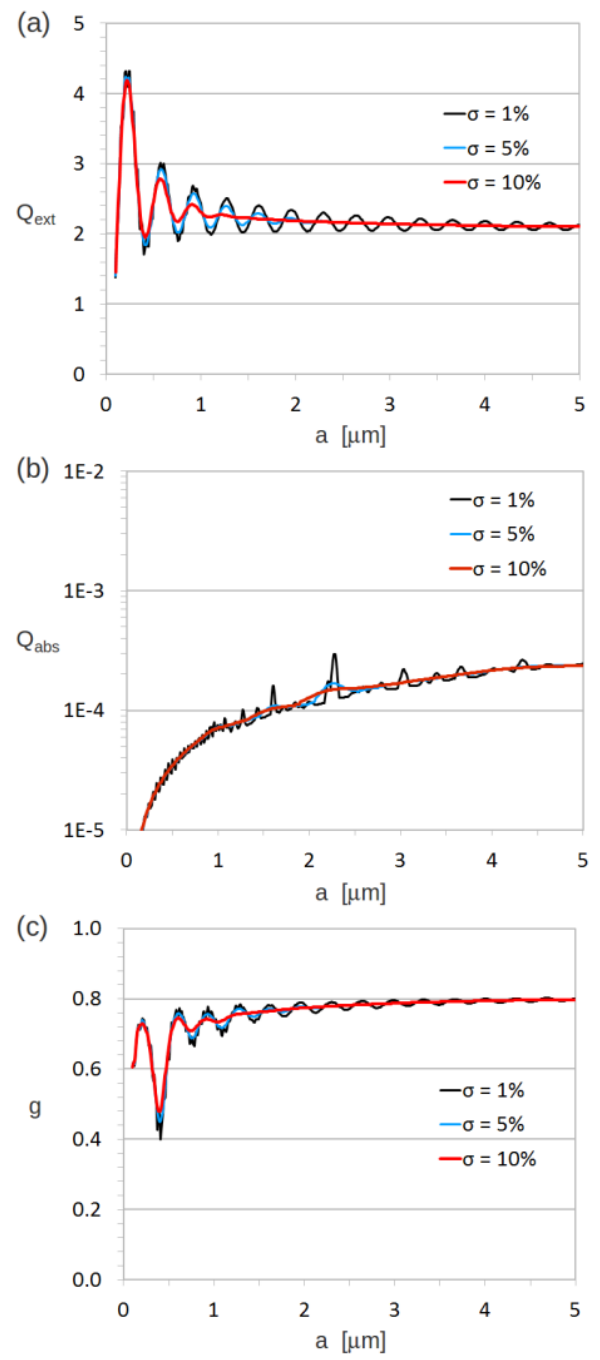

FIG. 5. Poly-disperse $(\sigma=1 \%, 5 \%, 10 \%)$ Mie theory calculations for GOS:Tb spheres (emission: $0.545 \mu \mathrm{m}$ ). Shown as a function of radius, $a$, are: (a) the extinction efficiency, $Q_{e x t}$ (b) the absorption efficiency, $Q_{a b s}$ and (c) the asymmetry factor, $g$. 
(a)
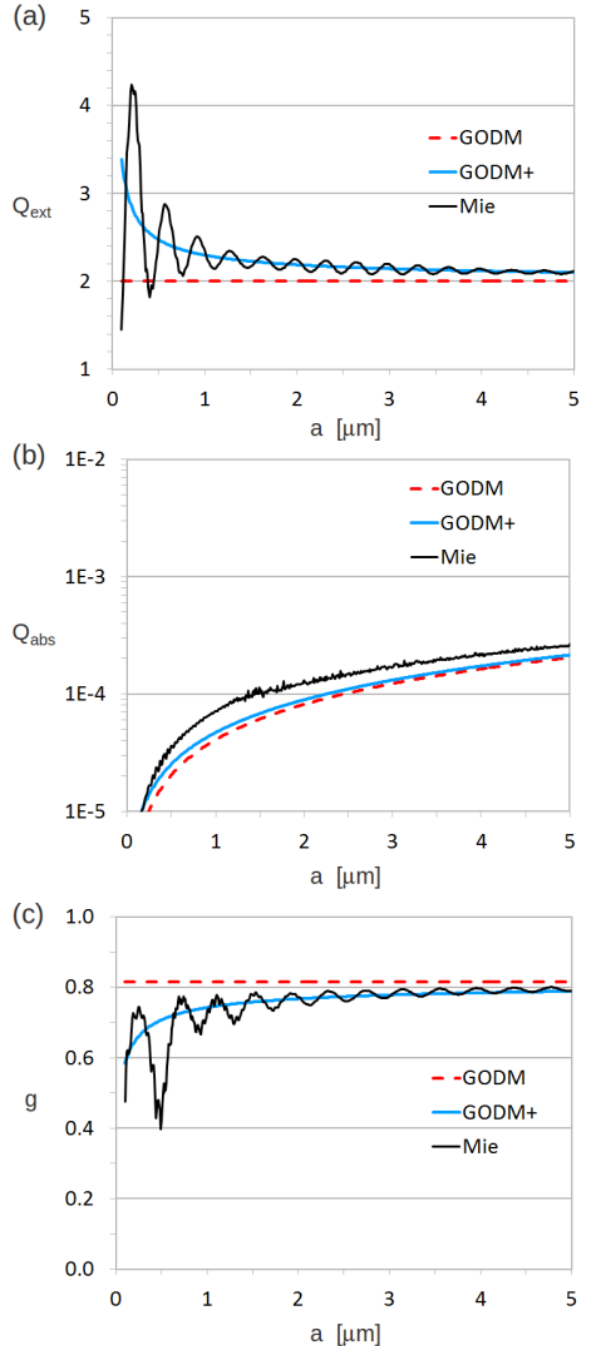

FIG. 6. Mie theory predictions averaged over emission spectrum and size-distribution $(\sigma=$ $1 \%)$ compared to and geometrical optics models (GODM, GODM+) for GOS:Tb spheres. Shown, as a function of radius, $a$, are: (a) the extinction efficiency, $Q_{e x t}(\mathrm{~b})$ the absorption efficiency, $Q_{a b s}$ and (c) the asymmetry factor, $g$. 

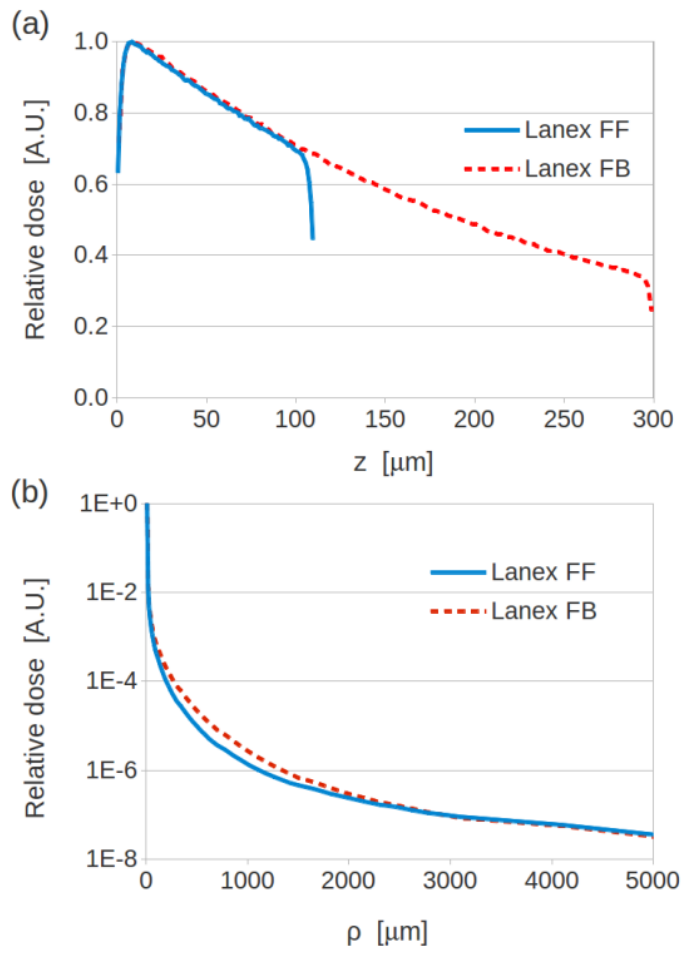

FIG. 7. (a) Depth and (b) radial distributions of dose deposited in the phosphor layer of the Lanex screens, as calculated using DOSRZnrc.

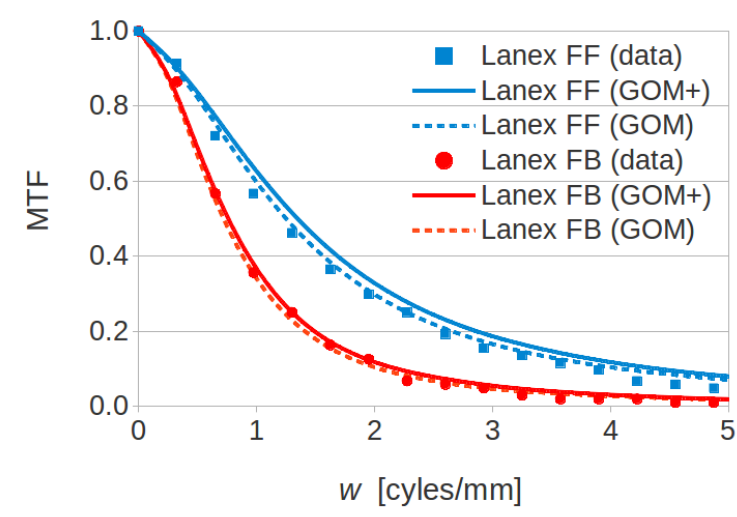

FIG. 8. Simulated MTFs for Lanex Fast Front and Fast Back screens as calculated using GODM (dashed lines) and GODM+ models (solid lines) in comparison to experimental data (data points).

\section{DISCUSSION}


The fine-structure and the slower oscillatory behavior of Mie predictions are known to reflect genuine physical effects. ${ }^{6}$ However, they are appropriate to a precise wavelength and a perfectly spherical particle of a precise radius. The effects would be expected to average out to varying extents in real phosphor screens, in which there are distributions of grain size, emission wavelength and indeed grain shape. Such averaging effects were demonstrated in Fig. 4 and Fig. 5. The modeler of optical transport in phosphor screens should therefore be skeptical about the relevance of any fine-structure or oscillations in the Mie theory predictions of transport parameters.

The results presented here in Fig. 6 suggest that the extended geometrical optics model (GODM+) provides accurate predictions of average-trend Mie values for $Q_{e x t}$ and $g$ (for grain radii $>1 \mu \mathrm{m}$ ). The $Q_{a b s}$ parameter predictions consistently underestimate Mie theory, but are likely to be adequate. Note that: (i) there are large uncertainties on the values of the imaginary components of phosphor RI anyway; (ii) the spread of optical photons is only weakly dependent on $Q_{a b s}$ and (iii) the accuracy of the Mie prediction can only be approximate anyway because of the assumption of spherical grains. The simpler GODM model also provides approximate agreement for the three transport parameters and may be quite adequate. This is supported in the MTF predictions for simulated screens in which both the GODM and GODM+ models provide substantially accurate agreement with experimental values.

The temptation to adjust the physical parameters (e.g. $a, n_{g}, z_{g}, n_{b}, p_{f}$ ) to further improve the agreement of simulated and measured MTFs further was resisted. It would be over-optimistic to think that all optical and x-ray processes were modeled accurately given the unknowns. Firstly, experimental errors and uncertainties are inevitable in the measurement of MTFs e.g. in extrapolation of line-spread-function tails. ${ }^{18}$ Secondly, there is uncertainty in the amount, composition and location of scattering material that was above and below the phosphor screens in experimental measurements. Thirdly, knowledge of the composition of the screens is incomplete. Even for the Lanex Fast Back screen, for which the most information was available, that information can be traced back to nominal values referenced as private communications from the manufacturer or inferred from patent 
documents. Fourthly, the structure of screens is more complicated than assumed. For example, typical screens, to varying degrees, contain residual air pockets in the phosphor layer. ${ }^{26}$ In view of these observations the agreement obtained in this study is remarkably encouraging.

In future work, the model presented here could be extended to take account of more complex screen structures, where detailed and specific information can be found. For example, the binder in some screens (although not Lanex Fast screens to the best of our knowledge) are doped with absorbing dye or carbon particles. ${ }^{27}$ The protective undercoat can also have more complicated optical properties than assumed here. ${ }^{16}$ An examination of the effects of grain shape and surface roughness on predictions would be worth pursuing. These deviations from the perfect spherical grain would be expected to have some quantitative impact on the values of transport parameters. Although there are no general analytical expressions available as in Mie theory when a scattering body is non-spherical, numerical techniques can be applied to solve the problem. ${ }^{28}$

\section{CONCLUSION}

The precise values of light transport parameters in powdered-phosphors, predicted in Mie-type calculations, are highly-sensitive to exact grain size and shape. The trend behaviors, beneath this numerical fine-structure, remain fairly simple and robust to deviations in grain shape and size and emission spectrum. For a realistic screen structure, with a distribution in both grain size and emission wavelength, only the average trend of Mie theory is relevant. This is adequately explained by simpler geometrical optics-based models.

\section{ACKNOWLEDGEMENTS}

This work was partially supported by research Grant No. C46/A10588 from Cancer Research UK. The authors acknowledge NIHR funding to the NHS Biomedical Research Center. 


\section{REFERENCES}

${ }^{1}$ M. Nik1, “Scintillation detectors for x-rays,” Meas. Sci. Technol. 17, R37-R54 (2006).

${ }^{2}$ G. G. Poludniowski and P. M. Evans, "Optical photon transport in powdered-phosphor scintllators.

Part1. Multiple scattering and validity of the Boltzmann transport equation.," submitted to Med. Phys. (2012).

${ }^{3}$ P. F. Liaparinos, I. S. Kandarakis, D. A. Cavouras, H. B. Delis, G. S. Panayiotakis, "Modeling granular phosphor screens by Monte Carlo methods,” Med Phys. 33(12), $4502-14$ (2006).

${ }^{4}$ T. Radcliffe, G. Barnea, B. Wowk, R. Rajapakshe, S. Shalev, "Monte Carlo optimization of metal/phosphor screens at megavoltage energies," Med Phys. 20(4), 1161-9 (1993).

${ }^{5}$ S. Pistrui-maximean, N. Freud, J. Letang, A. Koch, B. Munier, A. Walenta, G. Montarou, D. Babot, "Geant4 simulation of the response of phosphor screens for X-ray imaging," Nuclear Instruments and Methods in Physics Research A563(1), 196-199 (2006).

${ }^{6}$ H. C. van de Hulst, Light Scattering by Small Particles (Dover, New York, 1981).

${ }^{7}$ R. Morlotti, "X-ray efficiency and modulation transfer function of fluorescent Rare Earth screens, determined by the Monte Carlo method," J. Photographic Sci. 23, 181-189 (1975).

${ }^{8}$ P. F. Liaparinos, I. S. Kandarakis, D. A. Cavouras, H. B. Delis, G. S. Panayiotakis, "Monte Carlo study on the imaging performance of powder Lu2SiO5:Ce phosphor screens under x-ray excitation: comparison with Gd2O2S:Tb screens," Med Phys. 34(5), 1724-33 (2007).

${ }^{9}$ P. F. Liaparinos, I. S. Kandarakis, “The Monte Carlo evaluation of noise and resolution properties of granular phosphor screens,” Phys Med Biol. 54(4), 859-74 (2009).

${ }^{10}$ P. F. Liaparinos, I. S. Kandarakis, “The imaging performance of compact Lu2O3:Eu powdered phosphor screens: Monte Carlo simulation for applications in mammography," Med Phys. 36(6), 1985-97 (2009).

${ }^{11}$ M. K. Cho, H. K. Kim, T. Graeve, S. M Yun, C. H. Lim, H. Cho and J-M Kim, "Measurements of X-ray Imaging Performance of Granular Phosphors with Direct-Coupled CMOS Sensors," IEEE Transactions on Nuclear Science 55(3), 1338-1343 (2008).

${ }^{12} \mathrm{H}-\mathrm{H}$ Wang, P-W Lin, Y-C Wang and J-K Hu, “Composite optical film,” US Patent No. 2012/0008212A1 (2012).

$400{ }^{13}$ E.J. Popovici, L. Muresan, A. Hristea, E. Indrea, M. Vasilescu, M Nazarov and D. Young Jeon, "Synthesis and characterisation of rare earth oxysulphide phosphors.1.Studies on preparation of Gd2O2S:Tb Phosphor by the flux method," Optical Materials 27, 559-565 (2004).

${ }^{14}$ M. Gambaccini, A. Taibi, A Del Guerra, M. Marziani and A. Tuffanelli, "MTF evaluation of a phosphor-coated CCD for x-ray imaging," Phys. Med. Biol. 41, 2799-2806 (1996).

$405 \quad{ }^{15}$ R. E. Dickerson and P. C. Bunch, "Minimal crossover radiographic elements adapted for varied intensifying screen exposures," US Patent No. 5108881 (1992). 
${ }^{16}$ R. E. Dickerson, K. A. Duke and A. S. Fitterman, "Reflective radiographic material with incorporated developer," US Patent No. 7014977 (2006).

${ }^{17}$ H. Du, “Mie-scattering calculation,” Appl. Opt. 43. 1951-1956 (2004).

${ }^{18}$ S. Twomey and C. F. Bohren, "Simple approximations for calculations of absorption in clouds," J. Atmos. Science 37( 9), 2086-2094 (1980).

${ }^{19}$ A. A. Kokhanovsky, Light Scattering Media Optics: Problems and Solutions (Praxis Publishing Ltd, Chichester, UK, 2004).

${ }^{20}$ H. M. Nussenzveig and W. J. Wiscombe, "Efficiency factors in Mie scattering," Physical Review 415 Letters 45(18), 1490-1494 (1980).

${ }^{21}$ T. Graeve and G. P. Weckler, "High-resolution CMOS imaging detector," Proc. SPIE 4320, 68-76 (2001).

${ }^{22}$ S. M. Yun, M. K. Cho, C.H. Lim, H. K.Kim, T. Graeve, H Cho and J-M Kim, "Cascaded linearsystems analysis of CMOS flat-panel detectors for digital radiography," Advanced Nondestructive 420 Evaluation II, 804-809 (2007).

${ }^{23}$ D. W. O. Rogers, I. Kawrakow, J. P. Seuntjens, B. R. B. Walters and E. Maiegra-Hing, "NRC User Codes for EGSnrc," NRCC Report PIRS-702(revB).

${ }^{24}$ G. Poludniowski, G. Landry, F. DeBlois, P. M. Evans and F. Verhaegen, "SpekCalc: a program to calculate photon spectra from tungsten anode x-ray tubes," Phys. Med. Biol. 54, N433 (2009).

${ }^{25}$ S. N. Kasrova, N. G. Sultanova, C. D. Ivanov, I. D. Nikolov, “Analysis of the dispersion of optical plastic materials," Optical Materials 29, 1481-1490 (2007).

${ }^{26}$ J. A. Rowlands, “The physics of Computed Radiography,” Phys. Med. Biol. 47, R123-R166 (2002).

${ }^{27}$ R. E. Dickerson and P. C. Bunch, "Radiographic elements with selected speed relationships," US Patent No. 4997750 (1991). 